\title{
Size effect on competition of two diffusion mechanisms for drug molecules in amorphous polymers
}

\author{
Zhijian Zhao Qi Wang* Li Zhang \\ Department of Chemistry, Zhejiang University, Hangzhou 310027, P. R. China
}

\section{Supporting Information}

1. The parameters of the force field used in this simulation

POLYMER

van der Waals parameters

\begin{tabular}{cccccc}
\hline \hline Atom & $\mathrm{R} / \AA$ & $\varepsilon / \mathrm{kcal} \cdot \mathrm{mol}^{-1}$ & Atom & $\mathrm{R} / \AA$ & $\varepsilon / \mathrm{kcal} \cdot \mathrm{mol}^{-1}$ \\
\hline $\mathrm{C}$ & 1.85 & 0.12 & $\mathrm{CH}$ & 1.85 & 0.09 \\
$\mathrm{CH}_{3}$ & 2.00 & 0.15 & $\mathrm{CH}_{2}$ & 1.925 & 0.12 \\
$\mathrm{O}$ & 1.65 & 0.15 & $\mathrm{O}(=\mathrm{C})$ & 1.60 & 0.20 \\
\hline \hline
\end{tabular}

Bond stretching parameters

\begin{tabular}{cccccc}
\hline \hline Bond & $\mathrm{r} / \AA$ & $\mathrm{K}_{\mathrm{r}} / \mathrm{kcal} \cdot \mathrm{mol}^{-1} \cdot \AA^{2}$ & Bond & $\mathrm{r} / \AA$ & $\mathrm{K}_{\mathrm{r}} / \mathrm{kcal} \cdot \mathrm{mol}^{-1} \cdot \AA^{2}$ \\
\hline $\mathrm{C}-\mathrm{CH}$ & 1.444 & 317.0 & $\mathrm{C}-\mathrm{O}$ & 1.364 & 450.0 \\
$\mathrm{C}=\mathrm{O}$ & 1.229 & 570.0 & $\mathrm{CH}_{2}-\mathrm{CH}_{2}$ & 1.526 & 310.0 \\
$\mathrm{CH}-\mathrm{O}$ & 1.425 & 320.0 & $\mathrm{CH}_{-}-\mathrm{CH}_{3}$ & 1.526 & 310.0 \\
$\mathrm{CH}_{2}-\mathrm{O}$ & 1.410 & 320.0 & & & \\
\hline \hline
\end{tabular}

Angle bending parameters

\begin{tabular}{cccccc}
\hline \hline Angle & $\theta /{ }^{\circ}$ & $\mathrm{K}_{\theta} / \mathrm{kcal} \cdot \mathrm{mol}^{-1} \cdot \mathrm{rad}^{-2}$ & Angle & $\theta /{ }^{\circ}$ & $\mathrm{K}_{\theta} / \mathrm{kcal}^{\circ} \mathrm{mol}^{-1} \cdot \mathrm{rad}^{-2}$ \\
\hline $\mathrm{C}-\mathrm{CH}-\mathrm{O}$ & 110.3 & 60.0 & $\mathrm{CH}-\mathrm{C}=\mathrm{O}$ & 120.4 & 80.0 \\
$\mathrm{O}=\mathrm{C}-\mathrm{O}$ & 118.1 & 80.0 & $\mathrm{CH}_{3}-\mathrm{O}-\mathrm{C}$ & 116.8 & 60.0 \\
$\mathrm{C}-\mathrm{O}-\mathrm{CH}$ & 116.8 & 60.0 & $\mathrm{O}-\mathrm{CH}_{2}-\mathrm{CH}_{2}$ & 109.5 & 50.0 \\
$\mathrm{CH}-\mathrm{C}-\mathrm{O}$ & 120.4 & 80.0 & $\mathrm{C}-\mathrm{O}-\mathrm{CH}_{2}$ & 116.8 & 60.0 \\
$\mathrm{CH}_{3}-\mathrm{CH}-\mathrm{O}$ & 120.4 & 50.0 & $\mathrm{CH}_{2}-\mathrm{O}-\mathrm{CH}_{2}$ & 109.5 & 60.0 \\
$\mathrm{CH}_{3}-\mathrm{O}-\mathrm{CH}$ & 109.5 & 60.0 & $\mathrm{CH}-\mathrm{O}-\mathrm{CH}_{2}$ & 109.5 & 60.0 \\
$\mathrm{CH}_{3}-\mathrm{CH}-\mathrm{C}$ & 129.3 & 63.0 & $\mathrm{CH}_{3}-\mathrm{O}-\mathrm{CH}_{2}$ & 109.5 & 60.0 \\
\hline \hline
\end{tabular}


Torsional parameters

\begin{tabular}{|c|c|c|c|c|c|}
\hline Bond & $\mathrm{V}_{\mathrm{n}}\left(\mathrm{kcal} \cdot \mathrm{mol}^{-1}\right)$ & $\gamma /{ }^{\circ}$ & Bond & $\mathrm{V}_{\mathrm{n}}\left(\mathrm{kcal} \cdot \mathrm{mol}^{-1}\right)$ & $\gamma /{ }^{\circ}$ \\
\hline \multirow[t]{3}{*}{$\mathrm{X}-\mathrm{C}-\mathrm{CH}-\mathrm{X}$} & $\mathrm{V}_{1}=0.3$ & 0 & $\mathrm{X}-\mathrm{CH}_{2}-\mathrm{CH}_{2}-\mathrm{X}$ & $\mathrm{V}_{3}=4.0$ & 0 \\
\hline & $\mathrm{V}_{2}=2.1$ & 0 & $\mathrm{X}-\mathrm{CH}-\mathrm{O}-\mathrm{X}$ & $\mathrm{V}_{1}=2.2$ & 180 \\
\hline & $\mathrm{V}_{3}=0.4$ & 180 & & $\mathrm{~V}_{2}=2.7$ & 180 \\
\hline $\mathrm{X}-\mathrm{C}-\mathrm{O}-\mathrm{X}$ & $V_{2}=20.01$ & 180 & & $V_{3}=0.6$ & 180 \\
\hline \multirow[t]{2}{*}{$\mathrm{X}-\mathrm{CH}_{2}-\mathrm{O}-\mathrm{X}$} & $\mathrm{V}_{2}=0.1$ & 180 & & & \\
\hline & $V_{3}=0.38$ & 0 & & & \\
\hline
\end{tabular}

Electrostatic parameters ${ }^{\mathrm{a}}$

\begin{tabular}{cccccc}
\hline \hline Atom & Charge & Atom & Charge & Atom & Charge \\
\hline $\mathrm{C}$ & 0.798 & $\mathrm{CH}$ & 0.301 & $\mathrm{O}(=\mathrm{C})$ & -0.550 \\
$\mathrm{CH}_{3}$ & -0.076 & $\mathrm{CH}_{2}$ & 0.190 & $\mathrm{O}($ ester $)$ & -0.473 \\
$\mathrm{O}$ (aether) & -0.380 & & & & \\
\hline \hline
\end{tabular}

\section{BENZENE}

van der Waals parameters

\begin{tabular}{cccccc}
\hline \hline Atom & $\mathrm{R} / \AA$ & $\varepsilon / \mathrm{kcal} \cdot \mathrm{mol}^{-1}$ & Atom & $\mathrm{R} / \AA$ & $\varepsilon / \mathrm{kcal}^{\mathrm{mol}}{ }^{-1}$ \\
\hline $\mathrm{C}$ & 1.908 & 0.086 & $\mathrm{H}$ & 1.409 & 0.015 \\
\hline \hline
\end{tabular}

Bond stretching parameters

\begin{tabular}{cccccc}
\hline \hline Bond & $\mathrm{r} / \AA$ & $\mathrm{K}_{\mathrm{r}} / \mathrm{kcal} \cdot \mathrm{mol}^{-1} \cdot \AA^{2}$ & Bond & $\mathrm{r} / \AA$ & $\mathrm{K}_{\mathrm{r}} / \mathrm{kcal} \cdot \mathrm{mol}^{-1} \cdot \AA^{2}$ \\
\hline $\mathrm{C}-\mathrm{H}$ & 1.080 & 367.0 & $\mathrm{C}-\mathrm{C}$ & 1.400 & 469.0 \\
\hline \hline
\end{tabular}

Angle bending parameters

\begin{tabular}{|c|c|c|c|c|c|}
\hline "Angle & $\theta / /^{\circ}$ & $\mathrm{K}_{\theta} / \mathrm{kcal} \cdot \mathrm{mol}^{-1} \cdot \mathrm{rad}^{-2}$ & "Angle & $\theta / \circ$ & $\overline{\mathrm{C}} \mathrm{K}_{\theta} / \mathrm{kcal} \cdot \mathrm{mol}^{-1} \cdot \mathrm{rad}^{-2}$ \\
\hline C-C-H & 120.0 & 35.0 & C-C-C & 120.0 & 63.0 \\
\hline
\end{tabular}

Torsional parameters

\begin{tabular}{ccc}
\hline \hline Bond & $\mathrm{V}_{\mathrm{n}}\left(\mathrm{kcal} \cdot \mathrm{mol}^{-1}\right)$ & $\gamma /{ }^{\circ}$ \\
\hline $\mathrm{X}-\mathrm{C}-\mathrm{C}-\mathrm{X}$ & $\mathrm{V}_{2}=14.5$ & 180 \\
\hline \hline
\end{tabular}

Electrostatic parameters ${ }^{\text {a }}$

\begin{tabular}{cccc}
\hline \hline Atom & Charge & Atom & Charge \\
\hline $\mathrm{C}$ & -0.021 & $\mathrm{H}$ & 0.021 \\
\hline \hline
\end{tabular}




\section{ASPIRIN}

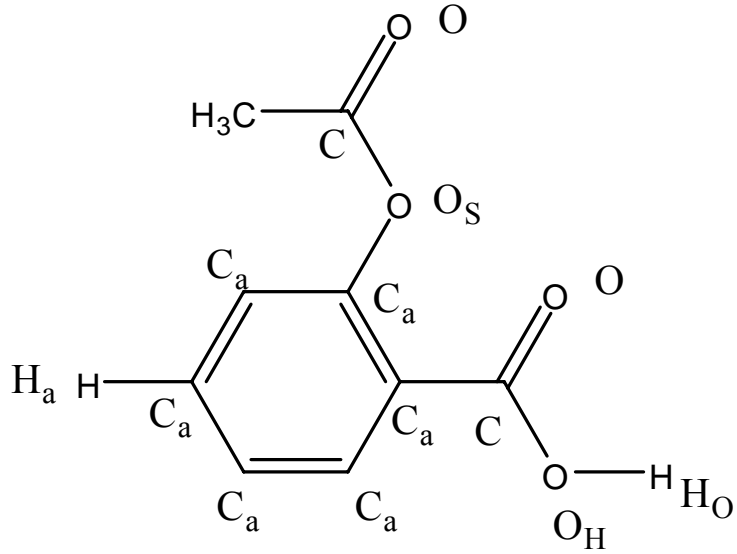

van der Waals parameters

\begin{tabular}{cccccc}
\hline \hline Atom & $\mathrm{R} / \AA$ & $\varepsilon / \mathrm{kcal}^{\mathrm{mol}} \mathrm{m}^{-1}$ & Atom & $\mathrm{R} / \AA$ & $\varepsilon / \mathrm{kcal}^{-\mathrm{mol}^{-1}}$ \\
\hline $\mathrm{C}_{\mathrm{a}}$ & 1.908 & 0.086 & $\mathrm{H}_{\mathrm{a}}$ & 1.409 & 0.015 \\
$\mathrm{C}$ & 1.908 & 0.086 & $\mathrm{O}$ & 1.661 & 0.210 \\
$\mathrm{O}_{\mathrm{H}}$ & 1.721 & 0.210 & $\mathrm{H}_{\mathrm{O}}$ & 0.0001 & 0.000 \\
$\mathrm{C}_{\mathrm{T}}$ & 1.908 & 0.109 & $\mathrm{H}_{1}$ & 1.387 & 0.016 \\
$\mathrm{O}_{\mathrm{S}}$ & 1.684 & 0.170 & & & \\
\hline \hline
\end{tabular}

Bond stretching parameters

\begin{tabular}{cccccc}
\hline \hline Bond & $\mathrm{r} / \AA$ & $\mathrm{K}_{\mathrm{r}} / \mathrm{kcal} \cdot \mathrm{mol}^{-1} \cdot \AA^{2}$ & Bond & $\mathrm{r} / \AA$ & $\mathrm{K}_{\mathrm{r}} / \mathrm{kcal} \cdot \mathrm{mol}^{-1} \cdot \AA^{2}$ \\
\hline $\mathrm{C}_{\mathrm{a}}-\mathrm{C}_{\mathrm{a}}$ & 1.400 & 469.0 & $\mathrm{C}_{\mathrm{a}}-\mathrm{H}_{\mathrm{a}}$ & 1.080 & 367.0 \\
$\mathrm{C}_{\mathrm{a}}-\mathrm{O}_{\mathrm{S}}$ & 1.373 & 372.4 & $\mathrm{C}_{\mathrm{a}}-\mathrm{C}$ & 1.409 & 469.0 \\
$\mathrm{C}-\mathrm{O}_{\mathrm{S}}$ & 1.343 & 411.3 & $\mathrm{C}-\mathrm{O}$ & 1.229 & 570.0 \\
$\mathrm{C}-\mathrm{C}_{\mathrm{T}}$ & 1.522 & 317.0 & $\mathrm{C}-\mathrm{O}_{\mathrm{H}}$ & 1.364 & 450.0 \\
$\mathrm{H}_{\mathrm{O}}-\mathrm{O}_{\mathrm{H}}$ & 0.960 & 553.0 & $\mathrm{H}_{1}-\mathrm{C}_{\mathrm{T}}$ & 1.090 & 340.0 \\
\hline \hline
\end{tabular}

Angle bending parameters

\begin{tabular}{|c|c|c|c|c|c|}
\hline Angle & $\theta / \circ$ & $\mathrm{K}_{\theta} / \mathrm{kcal} \cdot \mathrm{mol}^{-1} \cdot \mathrm{rad}^{-2}$ & Angle & $\theta / \circ$ & $\mathrm{K}_{\theta} / \mathrm{kcal} \cdot \mathrm{mol}^{-1} \cdot \mathrm{rad}^{-2}$ \\
\hline $\mathrm{C}_{\mathrm{a}}-\mathrm{C}_{\mathrm{a}}-\mathrm{C}_{\mathrm{a}}$ & 120.0 & 63.0 & $\mathrm{C}_{\mathrm{a}}-\mathrm{C}_{\mathrm{a}}-\mathrm{H}_{\mathrm{a}}$ & 120.0 & 35.0 \\
\hline $\mathrm{C}_{\mathrm{a}}-\mathrm{C}_{\mathrm{a}}-\mathrm{O}_{\mathrm{S}}$ & 119.2 & 69.8 & $\mathrm{C}_{\mathrm{a}}-\mathrm{C}_{\mathrm{a}}-\mathrm{C}$ & 120.0 & 63.0 \\
\hline $\mathrm{C}_{\mathrm{a}}-\mathrm{O}_{\mathrm{S}}-\mathrm{C}$ & 117.6 & 64.6 & $\mathrm{O}_{\mathrm{S}}-\mathrm{C}-\mathrm{O}$ & 122.4 & 76.2 \\
\hline $\mathrm{O}_{\mathrm{S}}-\mathrm{C}-\mathrm{C}_{\mathrm{T}}$ & 112.0 & 69.3 & $\mathrm{O}-\mathrm{C}-\mathrm{C}_{\mathrm{T}}$ & 120.4 & 80.0 \\
\hline $\mathrm{C}-\mathrm{C}_{\mathrm{T}}-\mathrm{H}_{1}$ & 109.5 & 50.0 & $\mathrm{H}_{1}-\mathrm{C}_{\mathrm{T}}-\mathrm{H}_{1}$ & 109.5 & 35.0 \\
\hline $\mathrm{C}_{\mathrm{a}}-\mathrm{C}-\mathrm{O}$ & 123.4 & 68.7 & $\mathrm{C}_{\mathrm{a}}-\mathrm{C}-\mathrm{O}_{\mathrm{H}}$ & 120.0 & 70.0 \\
\hline $\mathrm{O}-\mathrm{C}-\mathrm{O}_{\mathrm{H}}$ & 122.9 & 77.4 & $\mathrm{H}_{\mathrm{O}}-\mathrm{O}_{\mathrm{H}}-\mathrm{C}$ & 113.0 & 35.0 \\
\hline
\end{tabular}


Torsional parameters

\begin{tabular}{cccccc}
\hline \hline Bond & $\mathrm{V}_{\mathrm{n}}\left(\mathrm{kcal} \cdot \mathrm{mol}^{-1}\right)$ & $\gamma /{ }^{\circ}$ & Bond & $\mathrm{V}_{\mathrm{n}}\left(\mathrm{kcal} \cdot \mathrm{mol}^{-1}\right)$ & $\gamma /{ }^{\circ}$ \\
\hline $\mathrm{X}-\mathrm{C}_{\mathrm{a}}-\mathrm{C}_{\mathrm{a}}-\mathrm{X}$ & $\mathrm{V}_{2}=14.5$ & 180.0 & $\mathrm{X}-\mathrm{C}_{\mathrm{a}}-\mathrm{O}_{\mathrm{S}}-\mathrm{X}$ & $\mathrm{V}_{2}=1.8$ & 180.0 \\
$\mathrm{X}-\mathrm{C}_{\mathrm{a}}-\mathrm{C}-\mathrm{X}$ & $\mathrm{V}_{2}=14.5$ & 180.0 & $\mathrm{X}-\mathrm{C}-\mathrm{O}_{\mathrm{S}}-\mathrm{X}$ & $\mathrm{V}_{2}=5.4$ & 180.0 \\
$\mathrm{X}-\mathrm{C}_{\mathrm{T}}-\mathrm{C}-\mathrm{X}$ & $\mathrm{V}_{2}=0.0$ & 180.0 & $\mathrm{X}-\mathrm{C}-\mathrm{O}_{\mathrm{H}}-\mathrm{X}$ & $\mathrm{V}_{2}=4.6$ & 180.0 \\
\hline \hline
\end{tabular}

Electrostatic parameters ${ }^{\text {a }}$

\begin{tabular}{cccccc}
\hline \hline Atom & Charge & Atom & Charge & Atom & Charge \\
\hline $\mathrm{C}_{\mathrm{a}}$ & 0.075 & $\mathrm{H}_{\mathrm{a}}$ & 0.026 & $\mathrm{O}_{\mathrm{H}}$ & -0.164 \\
$\mathrm{C}$ & 0.590 & $\mathrm{H}_{\mathrm{l}}$ & 0.065 & $\mathrm{O}_{\mathrm{S}}$ & -0.050 \\
$\mathrm{C}_{\mathrm{T}}$ & -0.186 & $\mathrm{H}_{\mathrm{O}}$ & 0.214 & $\mathrm{O}$ & -0.650 \\
\hline \hline
\end{tabular}

\section{NIFEDIPINE}

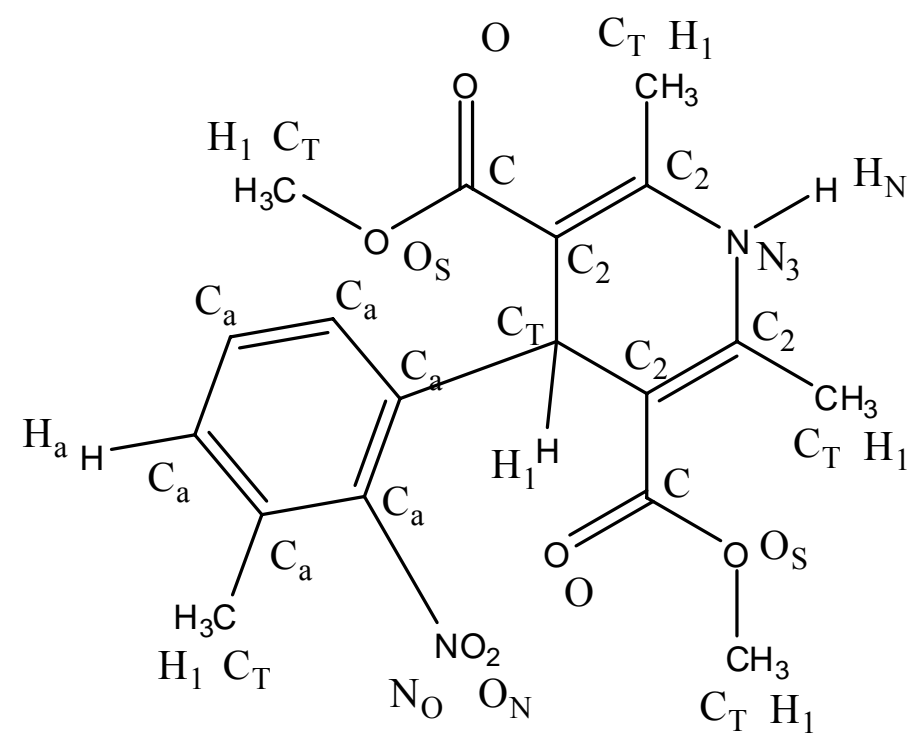

van der Waals parameters

\begin{tabular}{cccccc}
\hline \hline Atom & $\mathrm{R} / \AA$ & $\varepsilon / \mathrm{kcal} \cdot \mathrm{mol}^{-1}$ & Atom & $\mathrm{R} / \AA$ & $\varepsilon / \mathrm{kcal}^{-\mathrm{mol}^{-1}}$ \\
\hline $\mathrm{C}_{\mathrm{a}}$ & 1.908 & 0.086 & $\mathrm{H}_{\mathrm{a}}$ & 1.409 & 0.015 \\
$\mathrm{C}$ & 1.908 & 0.086 & $\mathrm{O}$ & 1.661 & 0.210 \\
$\mathrm{C}_{2}$ & 1.908 & 0.360 & $\mathrm{~N}_{\mathrm{O}}$ & 1.824 & 0.530 \\
$\mathrm{C}_{\mathrm{T}}$ & 1.908 & 0.109 & $\mathrm{H}_{1}$ & 1.387 & 0.016 \\
$\mathrm{O}_{\mathrm{S}}$ & 1.684 & 0.170 & $\mathrm{H}_{\mathrm{N}}$ & 0.600 & 0.016 \\
$\mathrm{~N}_{3}$ & 1.875 & 0.170 & & & \\
\hline \hline
\end{tabular}


Bond stretching parameters

\begin{tabular}{cccccc}
\hline \hline Bond & $\mathrm{r} / \AA$ & $\mathrm{K}_{\mathrm{r}} / \mathrm{kcal} \cdot \mathrm{mol}^{-1} \cdot \AA^{2}$ & Bond & $\mathrm{r} / \AA$ & $\mathrm{K}_{\mathrm{r}} / \mathrm{kcal} \cdot \mathrm{mol}^{-1} \cdot \AA^{2}$ \\
\hline $\mathrm{C}_{\mathrm{a}}-\mathrm{C}_{\mathrm{a}}$ & 1.400 & 469.0 & $\mathrm{C}_{\mathrm{a}}-\mathrm{H}_{\mathrm{a}}$ & 1.080 & 367.0 \\
$\mathrm{C}_{\mathrm{a}}-\mathrm{C}_{\mathrm{T}}$ & 1.510 & 317.0 & $\mathrm{C}_{\mathrm{a}}-\mathrm{N}_{\mathrm{O}}$ & 1.468 & 322.6 \\
$\mathrm{C}_{2}-\mathrm{C}_{\mathrm{T}}$ & 1.508 & 328.3 & $\mathrm{C}_{2}-\mathrm{C}_{2}$ & 1.324 & 589.7 \\
$\mathrm{C}_{2}-\mathrm{C}$ & 1.406 & 449.9 & $\mathrm{C}_{2}-\mathrm{N}_{3}$ & 1.340 & 486.3 \\
$\mathrm{C}_{\mathrm{T}}-\mathrm{O}_{\mathrm{S}}$ & 1.410 & 320.0 & $\mathrm{~N}_{3}-\mathrm{H}_{\mathrm{N}}$ & 1.010 & 434.0 \\
$\mathrm{C}-\mathrm{O}_{\mathrm{S}}$ & 1.343 & 411.3 & $\mathrm{C}-\mathrm{O}$ & 1.229 & 570.0 \\
$\mathrm{~N}_{\mathrm{O}}-\mathrm{O}_{\mathrm{N}}$ & 1.219 & 761.2 & $\mathrm{H}_{1}-\mathrm{C}_{\mathrm{T}}$ & 1.090 & 340.0 \\
\hline \hline
\end{tabular}

Angle bending parameters

\begin{tabular}{|c|c|c|c|c|c|}
\hline Angle & $\theta / \circ$ & $\mathrm{K}_{\theta} / \mathrm{kcal} \cdot \mathrm{mol}^{-1} \cdot \mathrm{rad}^{-2}$ & Angle & $\theta / \circ$ & $\mathrm{K}_{\theta} / \mathrm{kcal} \cdot \mathrm{mol}^{-1} \cdot \mathrm{rad}^{-2}$ \\
\hline $\mathrm{C}_{\mathrm{a}}-\mathrm{C}_{\mathrm{a}}-\mathrm{C}_{\mathrm{a}}$ & 120.0 & 63.0 & $\mathrm{C}_{\mathrm{a}}-\mathrm{C}_{\mathrm{a}}-\mathrm{H}_{\mathrm{a}}$ & 120.0 & 35.0 \\
\hline $\mathrm{C}_{\mathrm{a}}-\mathrm{C}_{\mathrm{a}}-\mathrm{C}_{\mathrm{T}}$ & 120.0 & 70.0 & $\mathrm{C}_{\mathrm{a}}-\mathrm{C}_{\mathrm{a}}-\mathrm{N}_{\mathrm{O}}$ & 119.5 & 66.9 \\
\hline $\mathrm{C}_{\mathrm{a}}-\mathrm{N}_{\mathrm{O}}-\mathrm{O}_{\mathrm{N}}$ & 118.1 & 68.7 & $\mathrm{O}_{\mathrm{N}}-\mathrm{N}_{\mathrm{O}}-\mathrm{O}_{\mathrm{N}}$ & 127.6 & 76.4 \\
\hline $\mathrm{C}_{\mathrm{a}}-\mathrm{C}_{\mathrm{T}}-\mathrm{C}_{2}$ & 109.7 & 64.6 & $\mathrm{C}_{\mathrm{a}}-\mathrm{C}_{\mathrm{T}}-\mathrm{H}_{1}$ & 111.0 & 46.8 \\
\hline $\mathrm{C}_{2}-\mathrm{C}_{\mathrm{T}}-\mathrm{C}_{2}$ & 109.7 & 64.6 & $\mathrm{C}_{2}-\mathrm{C}_{\mathrm{T}}-\mathrm{H}_{1}$ & 106.0 & 49.1 \\
\hline $\mathrm{C}_{\mathrm{T}}-\mathrm{C}_{2}-\mathrm{C}_{2}$ & 123.4 & 64.3 & $\mathrm{C}_{\mathrm{T}}-\mathrm{C}_{2}-\mathrm{C}$ & 119.7 & 63.9 \\
\hline $\mathrm{C}-\mathrm{C}_{2}-\mathrm{C}_{2}$ & 120.7 & 67.9 & $\mathrm{~N}_{3}-\mathrm{C}_{2}-\mathrm{C}_{2}$ & 124.6 & 70.3 \\
\hline $\mathrm{N}_{3}-\mathrm{C}_{2}-\mathrm{C}_{\mathrm{T}}$ & 122.2 & 67.0 & $\mathrm{C}_{2}-\mathrm{N}_{3}-\mathrm{C}_{2}$ & 124.7 & 66.2 \\
\hline $\mathrm{C}_{2}-\mathrm{N}_{3}-\mathrm{H}_{\mathrm{N}}$ & 119.4 & 49.1 & $\mathrm{O}-\mathrm{C}-\mathrm{C}_{2}$ & 119.1 & 72.8 \\
\hline $\mathrm{O}_{\mathrm{S}}-\mathrm{C}-\mathrm{C}_{2}$ & 119.1 & 72.8 & $\mathrm{O}_{\mathrm{S}}-\mathrm{C}-\mathrm{O}$ & 122.4 & 76.2 \\
\hline $\mathrm{C}_{\mathrm{T}}-\mathrm{O}_{\mathrm{S}}-\mathrm{C}$ & 115.1 & 63.6 & $\mathrm{H}_{1}-\mathrm{C}_{\mathrm{T}}-\mathrm{H}_{1}$ & 109.5 & 35.0 \\
\hline $\mathrm{O}_{\mathrm{S}}-\mathrm{C}_{\mathrm{T}}-\mathrm{H}_{1}$ & 108.8 & 50.8 & & & \\
\hline
\end{tabular}

Torsional parameters

\begin{tabular}{cccccc}
\hline \hline Bond & $\mathrm{V}_{\mathrm{n}}\left(\mathrm{kcal} \cdot \mathrm{mol}^{-1}\right)$ & $\gamma /{ }^{\circ}$ & Bond & $\mathrm{V}_{\mathrm{n}}\left(\mathrm{kcal} \cdot \mathrm{mol}^{-1}\right)$ & $\gamma /{ }^{\circ}$ \\
\hline $\mathrm{X}-\mathrm{C}_{\mathrm{a}}-\mathrm{C}_{\mathrm{a}}-\mathrm{X}$ & $\mathrm{V}_{2}=14.5$ & 180.0 & $\mathrm{X}-\mathrm{C}_{\mathrm{T}}-\mathrm{O}_{\mathrm{S}}-\mathrm{X}$ & $\mathrm{V}_{3}=1.15$ & 0.0 \\
$\mathrm{X}-\mathrm{C}_{\mathrm{a}}-\mathrm{C}_{\mathrm{T}}-\mathrm{X}$ & $\mathrm{V}_{2}=0.0$ & 0.0 & $\mathrm{X}-\mathrm{C}_{\mathrm{a}}-\mathrm{N}_{\mathrm{O}}-\mathrm{X}$ & $\mathrm{V}_{2}=2.4$ & 180.0 \\
$\mathrm{X}-\mathrm{C}_{2}-\mathrm{C}_{\mathrm{T}}-\mathrm{X}$ & $\mathrm{V}_{2}=0.0$ & 0.0 & $\mathrm{X}-\mathrm{C}_{2}-\mathrm{C}_{2}-\mathrm{X}$ & $\mathrm{V}_{2}=26.0$ & 180.0 \\
$\mathrm{X}-\mathrm{C}_{2}-\mathrm{C}-\mathrm{X}$ & $\mathrm{V}_{2}=8.7$ & 180.0 & $\mathrm{X}-\mathrm{C}-\mathrm{O}_{\mathrm{S}}-\mathrm{X}$ & $\mathrm{V}_{2}=5.4$ & 180.0 \\
$\mathrm{X}-\mathrm{C}_{2}-\mathrm{N}_{3}-\mathrm{X}$ & $\mathrm{V}_{2}=1.2$ & 180.0 & & & \\
\hline \hline
\end{tabular}

Electrostatic parameters ${ }^{\text {a }}$

\begin{tabular}{cccccc}
\hline \hline Atom & Charge & Atom & Charge & Atom & Charge \\
\hline $\mathrm{C}_{\mathrm{a}}$ & -0.016 & $\mathrm{H}_{\mathrm{a}}$ & 0.024 & $\mathrm{O}_{\mathrm{S}}$ & -0.101 \\
$\mathrm{C}$ & 0.543 & $\mathrm{H}_{\mathrm{N}}$ & 0.090 & $\mathrm{~N}_{3}$ & 0.279 \\
$\mathrm{C}_{2}$ & -0.043 & $\mathrm{H}_{1}$ & 0.037 & $\mathrm{~N}_{\mathrm{O}}$ & 1.230 \\
$\mathrm{C}_{\mathrm{T}}\left(\mathrm{H}_{3}-\mathrm{O}\right)$ & 0.075 & $\mathrm{O}(=\mathrm{C})$ & -0.703 & $\mathrm{O}(=\mathrm{N})$ & -0.628 \\
$\mathrm{C}_{\mathrm{T}}\left(\mathrm{H}_{3}-\mathrm{C}\right)$ & -0.144 & $\mathrm{C}_{\mathrm{T}}(\mathrm{H}-\mathrm{C})$ & -0.004 & & \\
\hline \hline
\end{tabular}

a: Charges in the unit of electrons 


\section{The log-log plots of MSD for penetrants in various polymer matrixes}

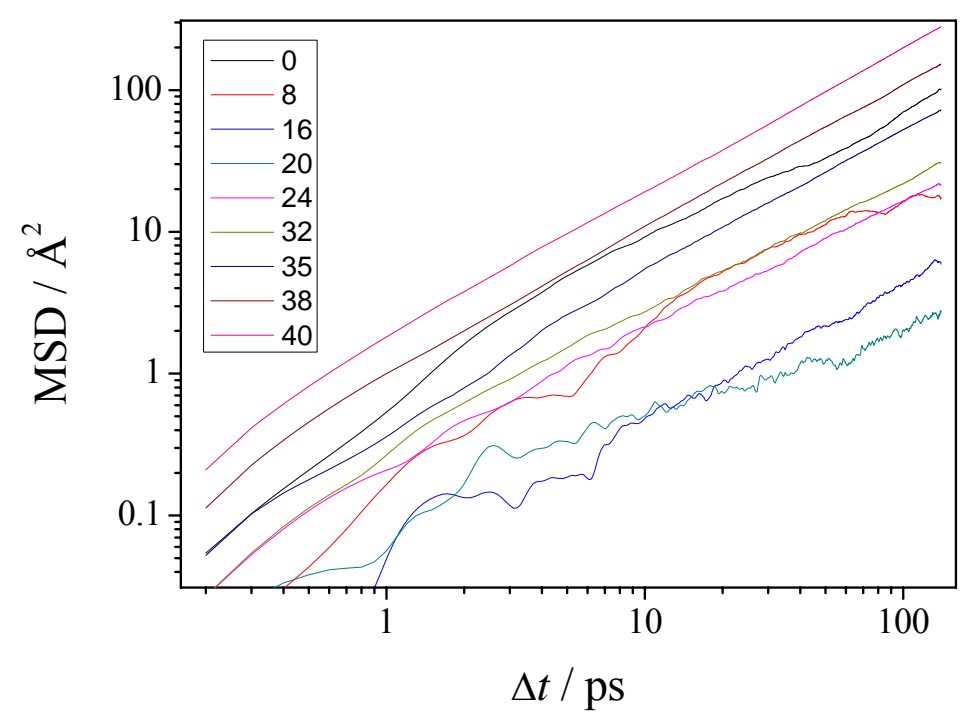

Figure S1. The log-log plots of MSD for benzene molecules in various polymer matrixes.

The label is the number of monomer ethylene glycol in the copolymer chain.

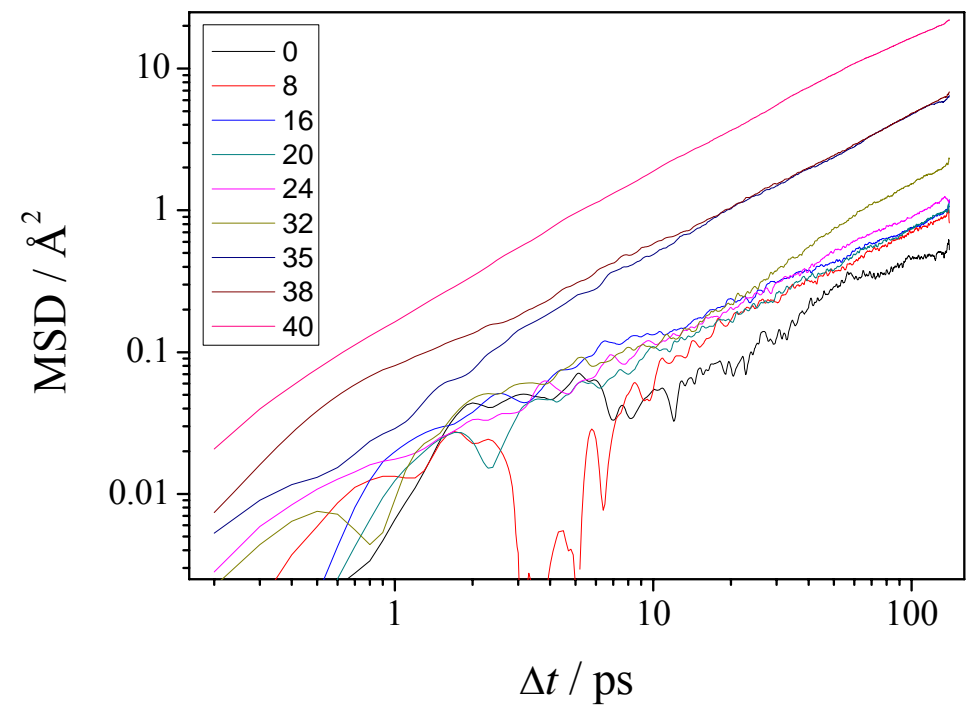

Figure S2. The log-log plots of MSD for aspirin molecules in various polymer matrixes.

The label is the number of monomer ethylene glycol in the copolymer chain. 


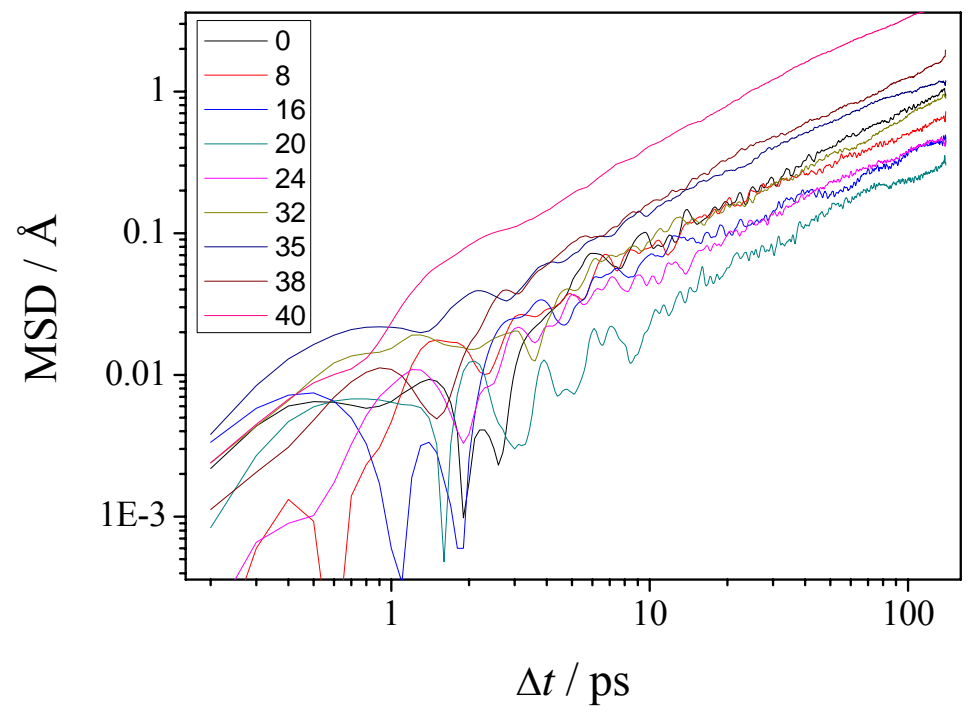

Figure S3. The log-log plots of MSD for nifedipine molecules in various polymer matrixes. The label is the number of monomer ethylene glycol in the copolymer chain. 